\title{
A Prospective Study On Impact Of Patient Counseling On The Management Of Risk Factors In Cardiovascular Diseases
}

\author{
, MahammadHaroon Shaik1*, Rajasekharreddy Polam2, Punnarao Bommaraboina2 \\ $, 1 *, 2$. Department of pharmacy practice, \\ A.M.Reddy Memorial College of Pharmacy, Narasaraopet- 522601, Guntur district, Andhra Pradesh.
}

*Address of correspondence:

Mahammad Haroon Shaik

Department of pharmacy practice,

A.M. Reddy Memorial College of pharmacy,

Narasaraopet- 522601, Guntur district,

Andhra Pradesh.

E-mail id: sharoon829@ gmail.com

DOI: 10.29322/IJSRP.11.01.2021.p10969

http://dx.doi.org/10.29322/IJSRP.11.01.2021.p10969

\begin{abstract}
:
Cardiovascular diseases are related to the heart and blood vessels. Aim: To assess the impact of patient counseling on the management of risk factors in cardiovascular diseases. Objectives: To analyze the impact of patient counseling in the prevention of disease progression and to assess the importance of medication adherence, lifestyle modifications in cardiovascular diseases.
\end{abstract}

Materials \& Methods: A prospective study with asample size of 120 patients is done in a tertiary care hospital at a period of six months. The sample includes age about $20-60$ years who were having risk factors of hypertension, diabetes, smoking, alcohol consumption, obesity, and a family history of cardiovascular diseases.

Results: A total $n=120(P<0.05)$ peoples were considered for this study, males $(n=85)$, females $(n=35)$. Department wise Inpatient $(n=61)$, outpatients $n=59$. The sample consists Ischaemic Disease $14.1 \%(n=17)$, Refractory Cardiac failure $13.3 \%(n=$ 16), Myocardial Infarction 33.3\% ( $\mathrm{n}=40)$, Coronary artery disease $19.1 \%(\mathrm{n}=23)$, Angina $10.8 \%(\mathrm{n}=13)$, others $9.1 \%(\mathrm{n}=11)$ and risk factors of HTN ( $n=18)$, DM ( $n=11)$, Smoking ( $n=18)$, Smoking+ alcohol $(n=25), H T N+$ DM $(n=30)$, Alcohol $(n=8)$, Alcohol+ DM ( $(n=3)$, HTN+ Smoking $(n=5)$, others $(n=3)$. Counselling was done to all patients; medication adherence assessment was done before and after counselling. A total of $75.8 \%(\mathrm{n}=91)$ shown to be improved and aware about medication adherence, risk factors management after patient counselling and about $24.1 \%(n=29)$ not improved.

KEYWORDS: Cardiovascular diseases, risk factors, patient counseling, medication adherence, management.

\section{INTRODUCTION:}

A cardiovascular disease is a group of diseases involving the heart and blood vessels. Cardiovascular disease includes coronary artery disease, angina, myocardial infarction, valvular heart disease, cardiomyopathy, arrhythmia, congenital heart disease, peripheral artery disease, and venous thrombosis $(1,3)$. Cardiovascular diseases are known to be preventable. Most cardiovascular diseases can be prevented by managing risk factors such as a healthy diet and physical activity, maintains normal blood pressure, normalize blood sugar levels, and maintain a healthy weight (2.13). Cardiovascular diseases are the major cause of mortality, morbidity, and, the most common cause of death in the world (4). Before the year 1900 malnutrition, infectious diseases are the most common cause of cardiovascular diseases. Now a day's cardiovascular diseases account for approximately $30 \%$ of death worldwide which includes nearly $40 \%$ in high-income countries $28 \%$ in low and middle-income countries, worldwide, 17.9 million people died from CVD in 2016 representing $31 \%$ of all global deaths of these, $85 \%$ are due to stroke and heart attack. 1.3 billion People smoked in the year 2003, a number that is projected to increase to 1.6 billion by the year 2030 . Currently, tobacco causes about 5 million deaths (9\%) of all deaths annually (3). Approximately 1.6 million are related to cardiovascular diseases. If the current pattern will continue by the year 2030 the death rate was increased to 10 million yearly (5). 
About $10 \%$ of Myocardial infarctions occur at the age of 40 years and $45 \%$ occur age 60 years. According to the American heart association, 81,100,000 American adults have one or more types of cardiovascular diseases (CVD) based on the data from 2003 to 2006 (6). In India, several studies show that the prevalence of cardiovascular diseases in the urban population is $11 \%$ and in the rural population is $7 \%(7)$.

ESTIMATED MORBIDITY RELATED TO HEART DISEASE 2010 -2030

\begin{tabular}{lcc}
\hline $\begin{array}{l}\text { ESTIMATED MORBIDITY RELATED TO } \\
\text { HEART DISEASE } 2010-2030 \text { DEATHS }\end{array}$ & BY 2010 & BY 2030 \\
\hline CVD deaths: Annual number of deaths & 18.1 million & 24.2 million \\
\hline CVD Deaths: percentage of all deaths & $30.8 \%$ & $32.5 \%$ \\
\hline CHD Deaths: percentage of all male deaths & $13.1 \%$ & $13.9 \%$ \\
CHD Deaths: percentage of all female deaths & $13.6 \%$ & Table-
\end{tabular}

\section{Estimated morbidity related to heart disease 2010-2030}

WHO estimates that cigarette smoking kills about 6 million people every year, of which 5 million are smokers and Tobacco is a known or probable cause of at least 25 diseases. The preventive measure is managing the cause and predisposition factors for the disease, lifestyle modification, and well- being of individuals (8). The European Society of Cardiology guideline recommends that pharmacological management is to reduce mortality, morbidity and improve patient quality of life whereas non-pharmacological management (self-care, behavioral and patient education) is to improve adherence to treatment and quality of life (9). Self- care is mainly helpful for the prevention and management of chronic illness (10). Poor medication adherence is a major problem in cardiovascular diseases this is due to limit care co-ordination, forgetfulness of the patient, confusion of treatment regimen, impaired cognitive behavior of the patient, poor communication between provider and patients, etc. (11). Cardiac rehabilitation is recommended for patients with heart failure, revascularization, and stable coronary artery disease (12). A traditional pharmacist activity like patient education, counseling about the disease, medication, and lifestyle modifications help to improve patient knowledge, medication adherence and preserves the health status of patients (14). This study is to assess the impact of patient counseling on the management of risk factors in cardiovascular diseases.

\section{MATERIALS AND METHODS:}

This was a prospective study carried at a tertiary care hospital with a duration of six months. The aim of this study is to assess the impact of patient counseling for the management of risk factors in cardiovascular diseases, and objectives are to improve and maintain patient functional capacity and well-being by managing and controlling risk factors, to analyze the impact of patient counseling in the prevention of disease progression, to assess the importance of medication adherence and lifestyle modifications in cardiovascular diseases. The sample size is $n=120$, study criteria includes

- $\quad$ Patients with the age of 20 to 60 years.

- Patients with alcohol and smoking.

- Patients with hypertension and diabetes

- Patients of obese.

- Patients with a family history of cardiovascular diseases

And excludes

- Patients with the age of fewer than 20 years and more than 60 years

- Patients with thyroid disorders

- Patients with cerebrovascular diseases

- Other comorbid disease conditions

Data is collected from the hospital by interviewing the patient, the entire session was carried out in private to create a favorable environment for information and provide education. Each session should be a period of approximately 10 to 15 minutes. Patients with cardiovascular diseases associated with risk factors. A data collection form was formulated which provides information about patient demographics (age, sex, case history, lab data, diagnosis, therapeutic management). Patient medication adherence questionnaire form prepared to assess the medication adherence, Counselling was done by using patient counseling leaflets in both regional language and English to prevent disease progression and to improve patient outcomes. 
The statistical analysis used for the study is descriptive statistics are range, mean, coefficient of variation, standard deviation calculated to age versus a number of patients, risk factors versus a number of patients, disease versus a number of patients. Zscore was calculated for the study population and probability values, skewness, kurtosis are calculated for study data. Medication adherence was assessed and two-way ANOVA is done on the study population before and after patient counseling. The statistical analysis is done by using Graphpad prism 8.3.1. (549) Software.

\section{RESULTS AND DISCUSSION:}

\section{Distribution of participants:}

The sample consists of a total 120 of peoples, among that $n=61(50.8 \%)$ peoples belong to the inpatient department, $n=59$ $(49.1 \%)$ peoples belong to the out-patient department. Figure 1 shown the department wise distribution of population

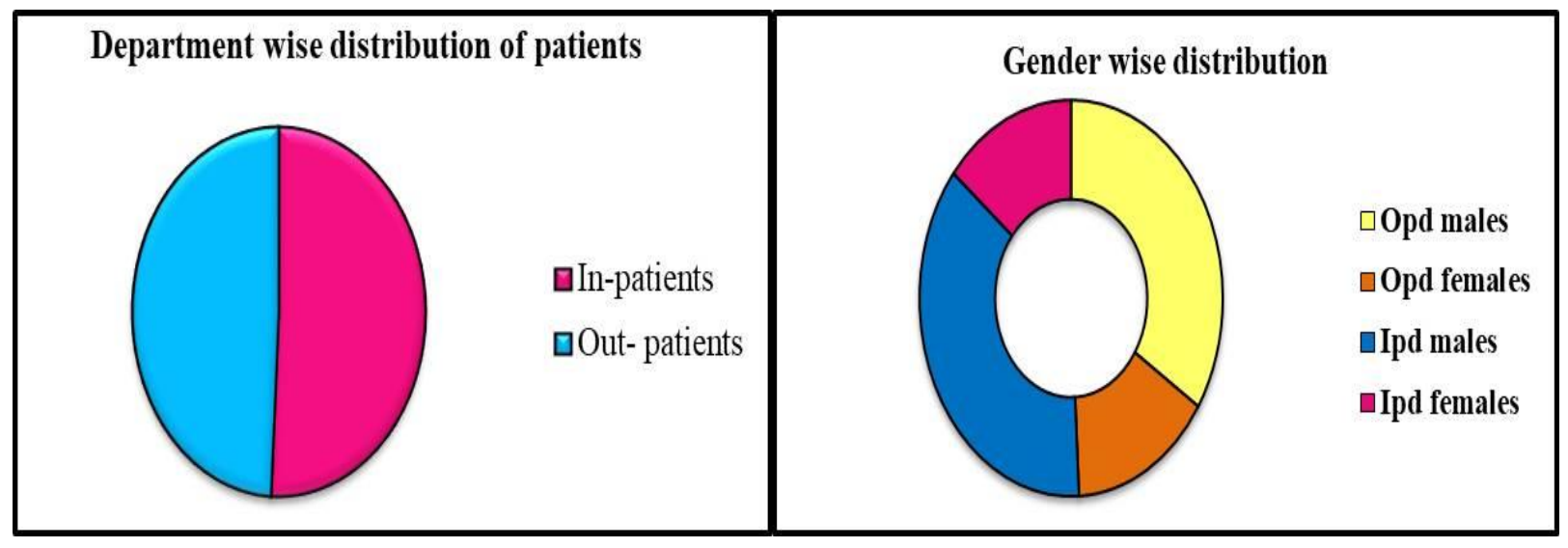

Figure-1.Department wise distribution, Figure-2 Gender wise distribution of Patients.of Patients.

Gender wise includes males $n=85$ (Inpatients-44, Outpatients- 41), whereas females $n=35$ (Inpatients- 19, Outpatients-18). Figure 2Shown the gender wise distribution of patients.

\section{Age-based demographics of cardiovascular disease patients:}

Age of 20 to 60 years is considered for this study, the collected data was distributed from the age of 25 to 30 years with a range of five. The below table indicates the age-wise distribution of cardiovascular disease patients and Figure 5 explains the age-wise demographics of patients

\begin{tabular}{cccccccc}
\hline Age range & $\mathbf{2 5}-\mathbf{3 0}$ & $\mathbf{3 1 - 3 5}$ & $\mathbf{3 6 - 4 0}$ & $\mathbf{4 1 - 4 5}$ & $\mathbf{4 6 - 5 0}$ & $\mathbf{5 1 - 5 5}$ & $\mathbf{5 6 - 6 0}$ \\
\hline Number of patients (n) & 2 & 7 & 9 & 18 & 21 & 38 & 25 \\
Percentage & $1.6 \%$ & $5.8 \%$ & $7.5 \%$ & $15 \%$ & $17.5 \%$ & $31.6 \%$ & $20.8 \%$
\end{tabular}

Table- 02. 


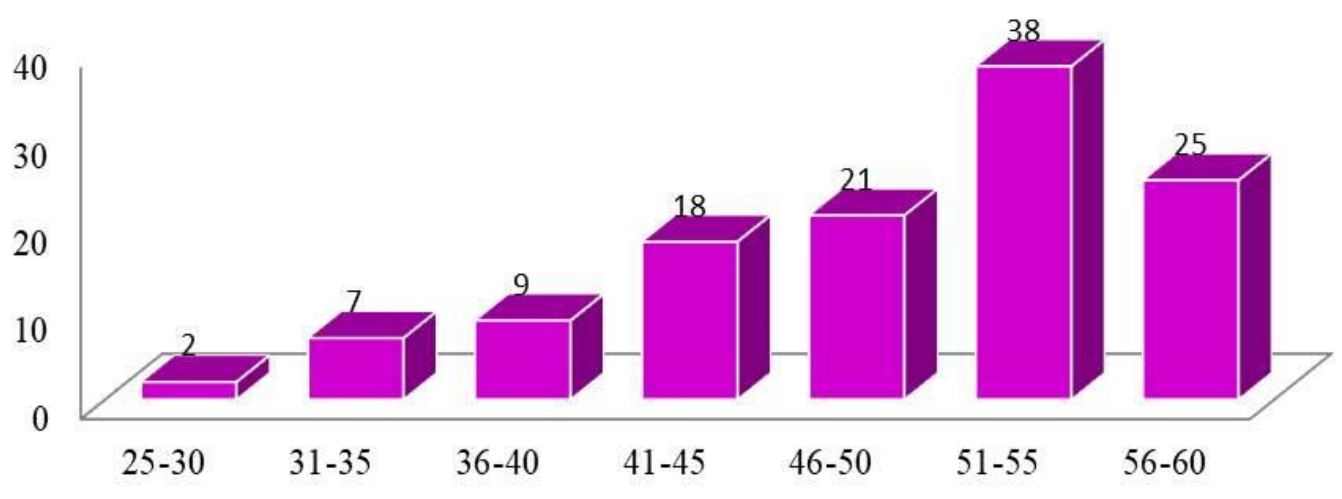

- No. of patients

Figure-3. Age-

wise distribution of cardiovascular disease patients

The above data was having a standard deviation of 12.32 , the Coefficient of variation is $71.87 \%$, the standard error of mean is 4.657, skewness value is 0.5689 , kurtosis value is -0.1182 , and probability is significant $(\mathrm{P}<0.05)$.

\section{Disease wise distribution of cardiovascular disease patients:}

Among 120 patients ischemic heart disease $14.1 \%(\mathrm{n}=17)$, Refractory cardiac failure $13.3 \%(\mathrm{n}=16)$, Myocardial infarction $33.3 \%(n=20)$, Coronary artery disease $19.1 \%(n=23)$, Angina pectoris $10.8 \%(n=13)$, others $9.1 \%(n=11)$ which includes complete heart blocks, dilated cardiomyopathy, pulmonary artery hypertension, deep vein thrombosis. The disease wise distribution of cardiovascular diseases has standard deviation of 10.62, standard error of mean is 4.336 , coefficient of variation is $53.10 \%$, skewness value is 1.719 , kurtosis value is 0.1182 , and probability is significant $(\mathrm{P}<0.05)$.

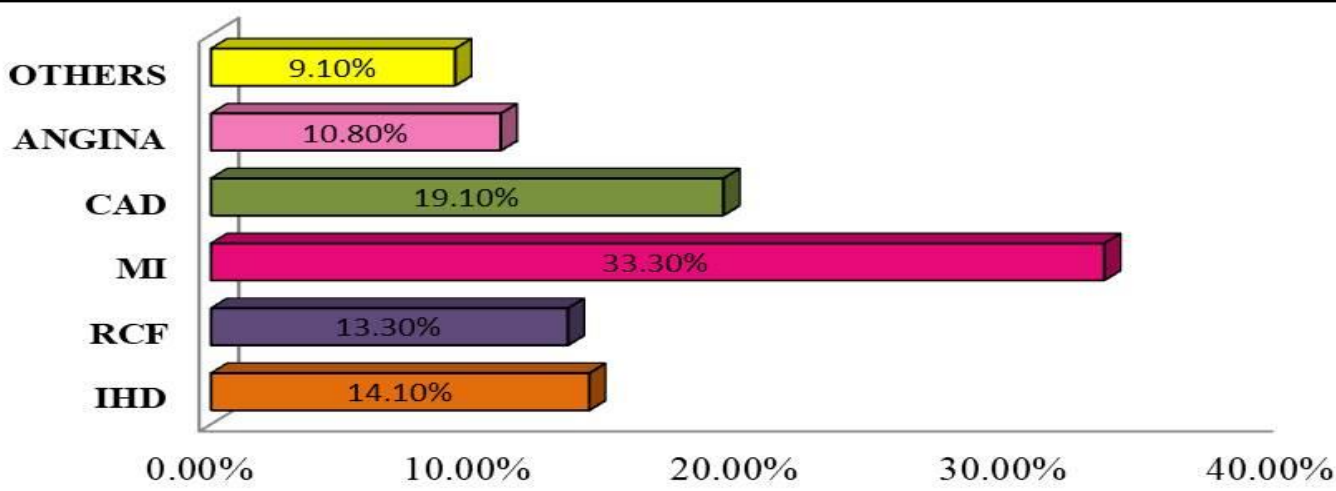

口IHD $\square$ RCF $\square$ MI $\square$ CAD $\square$ ANGINA $\square$ OTHERS

Figure -4. Disease wise distribution of patients

\section{Risk factor wise distribution:}

Out of total $(\mathrm{n}=120)$ patients risk factors are Diabetes $9.1 \%(\mathrm{n}=11)$, Hypertension $15 \%(\mathrm{n}=18)$, smoking $15 \%(\mathrm{n}=18)$, Smoking+ Alcohol 20.8\% $(n=25)$, Hypertension with Diabetes 25\% $(n=30)$, Alcoholics 6.6\% ( $n=8)$, Alcohol with Diabetes $2.5 \%(n=3)$, Hypertension with smoking $4.1 \%(\mathrm{n}=5)$, others $2.5 \%(\mathrm{n}=3)$ which includes family history, obesity etc., the above data has standard deviation of 9.838 , co-efficient of variation $73.17 \%$, standard error of mean 3.279 , skewness value is -0.5424 , kurtosis value is -1.036 , and probability is significant $(\mathrm{P}, 0.05)$. Figure 7 gives the information of various types of risk factors in cardiovascular disease patients. 


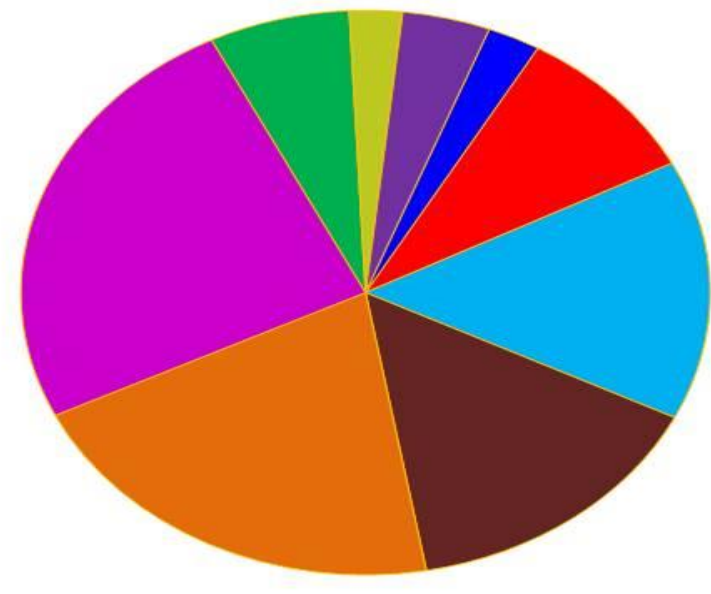

miabetes (9.1\%)

mypertension (15\%)

n Smoking ( 15\%)

Emoking + Alcohol (20.8\%)

$\because \mathbf{H T N}+$ DM $(25 \%)$

alcohol (6.6\%)

Alcohol + DM (2.5\%)

ETN + Smoking (4.1\%)

nothers $(2.5 \%)$

Figure-5. Distribution of patients based on risk factors

Medication adherence to cardiovascular disease patients:

Among total patients $(\mathrm{n}=120)$ medication adherence was assessed based on the data provided by the patient with the help of a self-prepared questionnaire. Data was splits into poor (0\% to 33\%), medium ( $34 \%$ to 66\%), high (67\%to 100\%) based on percentages. Before patient counseling 20.8\% $(n=25)$ has poor medication adherence, $58.3 \%(n=70)$ has medium medication adherence, $20.8 \%(n=25)$ has high medication adherence. After providing detailed information regarding the role of medication adherence to manage the risk factors improvement is observed. The below table 4 and figure 8,9 provide the information regarding medication adherence before after patient counseling.

\begin{tabular}{ccccccc}
\hline AGE & MEDICATION ADHERENCE BEFORE & \multicolumn{3}{c}{ MEDICATION ADHERENCE AFTER } \\
& \multicolumn{3}{c}{$\begin{array}{c}\text { POUNSELING } \\
\text { PATIENT COUNSELING }\end{array}$} \\
\hline $\mathbf{2 0 - 3 0}$ & Poor & Medium & High & Poor & Medium & High \\
$\mathbf{3 0 - 4 0}$ & 0 & 2 & 0 & 0 & 0 & 2 \\
$\mathbf{4 0 - 5 0}$ & 5 & 8 & 3 & 2 & 4 & 10 \\
$\mathbf{5 0 - 6 0}$ & 6 & 26 & 7 & 3 & 14 & 22 \\
Total & 14 & 34 & 15 & 8 & 20 & 35 \\
Percentage & 25 & 70 & 25 & 13 & 38 & 69 \\
\hline
\end{tabular}

Table-03. Level of medication adherence before and after counseling

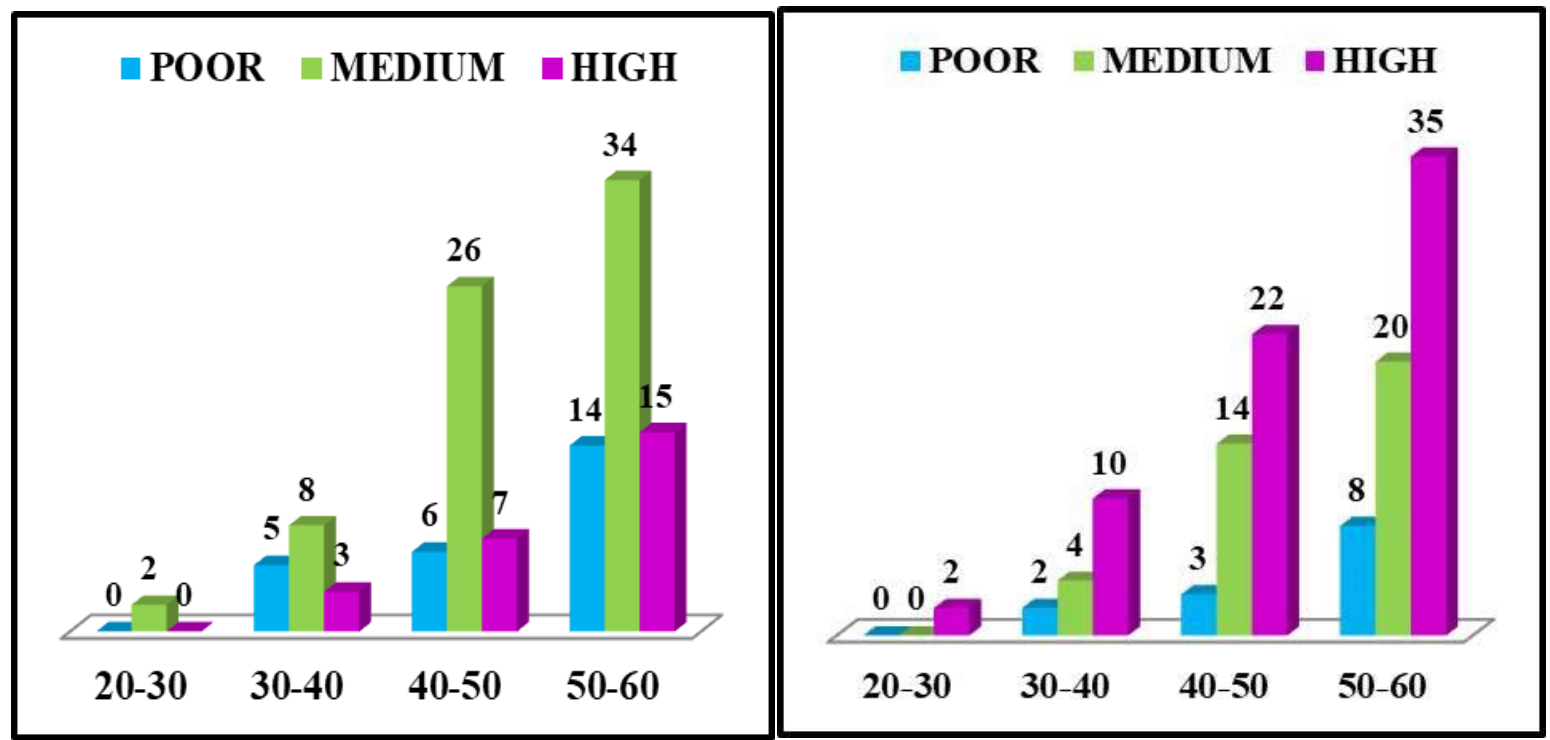

Figure-6.Medication adherence before 
Figure-7. Medication adherence after patient counselling patient counselling

\begin{tabular}{|c|c|c|c|c|c|c|c|c|c|c|}
\hline \multirow{2}{*}{$\begin{array}{l}\text { Anova } \\
\text { Table }\end{array}$} & \multicolumn{5}{|c|}{ BEFORE COUNSELING } & \multicolumn{5}{|c|}{ AFTER COUNSELING } \\
\hline & SS & DF & MS & $\mathrm{F}(\mathrm{DFn}, \mathrm{DFd})$ & $\mathrm{P}$ value & SS & DF & MS & $\begin{array}{l}\text { F(DFn, } \\
\text { DFd) }\end{array}$ & $\mathrm{P}$ value \\
\hline $\begin{array}{l}\text { Row } \\
\text { factor }\end{array}$ & 716.7 & 3 & 238.9 & $F(3,6)=7.713$ & 0.0176 & 716.7 & 3 & 238.9 & $\begin{array}{l}F(3,6)= \\
7.472\end{array}$ & 0.0189 \\
\hline $\begin{array}{l}\text { Column } \\
\text { factor }\end{array}$ & 337.5 & 2 & 168.8 & $F(2,6)=5.448$ & 0.0448 & 393.5 & 2 & 196.8 & $\begin{array}{l}F(2,6)= \\
6.154\end{array}$ & 0.0352 \\
\hline Residual & 185.8 & 6 & 30.7 & & & 191.8 & 6 & 31.97 & & \\
\hline
\end{tabular}

Table-04. Two way ANOVA of medication adherence before and after patient counselling

After the patient counselling medication adherence was improved. The level of medication adherence in patients after counselling Poor- $10.8 \%(n=13)$, Medium- 31.6\% ( $\mathrm{n}=38)$, High- 57.5\% ( $\mathrm{n}=69)$. Among total sample $(\mathrm{n}=120), 75.8 \%(\mathrm{n}=91)$ improved and $24.1 \%(n=29)$ not improved after patient counselling to cardiovascular disease patients.

\section{CONCLUSION:}

The main aim of the study is to evaluate the impact of patient counselling on the management of risk factors in cardiovascular diseases. This study gives information regarding the importance of medication adherence, lifestyle modifications to manage risk factors. Patients should have aware of their health this can be provided by the health care professionals. To manage risk factors medication adherence, lifestyle modifications, and medication adherence plays a major role in the prevention of disease progression. Medication non-adherence will worsen the risk factors which results in complications. In this current study patient counseling and education shows a good impact on participants for the management of risk factors.

\section{ACKNOWLEDGMENT:}

This thesis is the product of ample guidance, support, and encouragement from Ch. Gopala Krishna Assistant Professor (Department of Pharmacology)of A.M Reddy Memorial college of Pharmacy Narasaraopet, Guntur AP., and Chairman of Lalitha super-specialty hospitals, Guntur, AP. for providing me all facilities, throughout the research work.

\section{CONFLICT OF INTEREST:}

We declared that no conflict of interest.

\section{REFERENCES:}

1. Prevention of cardiovascular disease, Guidelines for assessment and management of cardiovascular risk: World health organization 2007 .

2. Textbook of best practices for cardiovascular disease prevention and programs; Centers for disease control and Prevention 2017.

3. Textbook of Harrison's Cardiovascular medicine; Joseph Loscaizo : 2010 page no. 33 to 37.

4. Tanmay Nag et al., Cardiovascular disease risk factors in Asian Indian population: A systematic review: journal of cardiovascular disease research 4(2013) 222-228.

5. Textbook of Pharmacotherapy A pathophysiologic approach; Joseph T. Dipiro; $8^{\text {th }}$ edition, page no. 55 to 273.

6. Textbook of clinical pharmacy and therapeutics; Roger Walker: $5^{\text {th }}$ edition, Page no. 312 to 354,376 to 380 .

7. Geevar Zachariah et al., Prevalence of Coronary artery disease and coronary risk factors in Kerala, South India: A Population survey- design and methods; ScienceDirect: doi.10.1016 ihj.2013.04.008.

8. G. Latorre et al., Counseling intervention to improve quality of life in patients with pre-existing Acute myocardial infarction (AMI) or chronic obstructive lung disease (COPD): A pilot study; JPREV-MEDHYG 2018; 59:E 153 -E 158.

9. Helene vailant Roussel et al., Patient education in chronic heart failure in primary care (ETIC) and its impact on patient quality of life; Design of a cluster-randomized trial; BMC family practice: (2014), 15:2008; DOI 10.1186/512875-014-0208-3.

10. Barbara Riegel et al., Self-Care for the prevention and management of cardiovascular disease And stroke; Journal of American Heart association: 2017;6: e006997. DOI: 10.1161/JAHA.117.006997.

11. Facts- A Tough pill to swallow, Medication adherence and cardiovascular diseases; American heart association, stroke association.

12. Long L et al., Exercise-based cardiac rehabilitation for adults with stable angina; Cochrane library DOI:n10.1002/14651858.CD012786.pub2.

13. Cardiovascular diseases the problem; World health organisation: http://www.who.int/healthinfo/globalburden disease/en http;//www.who.int/mediacentre/factsheets/fs317/en/index hmtl

14. Stefano Omboni et al., Effectiveness of pharmacist intervention in the management of cardiovascular diseases; Open Heart 2013:5:e00687, doi:10.1136/openhrt-2017-000687. 